\title{
DEEP THOUGHT VERSUS BYRNE
}

\begin{abstract}
The DT team
In July, 1989, GM Robert Byrne visited Carnegie-Mellon University and played a few friendly games against Deep Thought. Afterwards, Byrne arranged with the USA Today Sporting Network for a possible exhibition tele-match with Deep Thought to gather more material for an article that he is writing for New York Times. Meanwhile, the organizers of IJCAI (International Joint Conference on Artificial Intelligence) contacted the Deep Thought team for yet another exhibition match during the conference. We suggested to IJCAI that it might be possible to combine the two events. The IJCAI organizers promptly made the arrangements with USA Today and Byrne. A one-game match was scheduled for Aug. 23, 1:30-6:30 pm.
\end{abstract}

The color was determined before the match by the toss of a coin, and Deep Thought got White. We assumed that Byrne would play the French defence, and prepared the opening book accordingly. Byrne surprised us by playing Sicilian instead. By move 5, the machine was out of its tiny book. The machine, nonetheless, got some developmental advantages which lingered for the first half of the midgame. Byrne gradually neutralized DT's advantages and actually got the upper hand according to DT. But the machine managed to slip in 30. a4, 33. d5, and the tide slowly turned again. After 36. Nf1, DT suddenly realized that it was about to win a Pawn. Byrne apparently sensed the problem too and opted to sack a Knight for two Pawns which activated the Bishop on a8 and gave some counterplay. But the position became wide open, and DT was in its full element. Byme resigned on move 48.

After this match, a return game was arranged with USA Today Sporting Network for September 2, 1:00 pm. In this game DT played Black.

\author{
Deep Thought (2551) - GM Robert Byrne (2548) \\ USA TODAY Match, Game 1
}

1. e4 c5 2. c3 b6 3. d4 Bb7 4. Bd3 e6 5. Be3 Nf6 6. Nd2 Nc6 7. a3 d6 8. Qf3 g6 9. Ne2 Bg7 10. 0-0 0-0 11. b4 cxd4 12. cxd4 Qd7 13. Rac1 Rac8 14. h3 Ne7 15. Bg5 Rxc1 16. Rxc1 Ne8 17. Bb5 Qd8 18. Qg3 h6 19. Be3 d5 20. f3 Nd6 21. Bd3 b5 22. Rc5 a6 23. Bf4 Nc4 24. Bxc4 dxc4 25. Bd6 Re8 26. Rc7 Ba8 27. Rc5 Nc8 28. Be5 Bxe5 29. Qxe5 Nd6 30. a4 Qd7 31. Qf4 Kg7 32. h4 Bc6 33. d5 Ba8 34. dxe6 Rxe6 35. axb5 axb5 36. Nf1 Nxe4 37. fxe4 Rxe4 38. Qf2 c3 39. Nxc3 Rxb4 40. Ne3 Bc6 41. Ned5 Bxd5 42. Nxd5 Rb1+ 43. Kh2 Qd6+ 44 . g3 Qe5 45. Nc3 Qe1 46. Qd4+ Kh7 47. Nxb1 Qe2+ 48. Kg1 1-0.

GM Robert Byrne (2548) - Deep Thought (2551)

USA TODAY Match, Game 2

1. d4 d5 2. c4 dxc4 3. Nf3 Nf6 4. Qa4+ c6 5. Qxc4 Bf5 6. Nc3 Nbd7 7. g3 Nb6 8. Qb3 Be6 9. Qd1 Nbd5 10. Bg2 Nxc3 11. bxc3 Qa5 12. Qc2 Ne4 13. Bb2 Qf5 14. Qa4 a6 15. 0-0 Qh5 16. Rfe1 Qf5 17. c4 f6 18. Qb4 0-0-0 19. Rac1 Bf7 20. Qb6 g5 21. d5 c5 22. Bc3 e5 23. dxe6 Bxe6 24. Rb1 Rd7 25. Nd2 Nxc3 26. Bxb7+ Rxb7 27. Qc6+ Kd8 28. Rxb7 Bd7 29. Rxd7+ Qxd7 30. Qxf6+ Ke8 31. Qxc3 Bg7 32. Qe3+ Qe7 33. Ne4 Bd4 34. Qd3 Rf8 35. e3 Be5 36. Qd5 Rf7 37. Nxc5 Qd6 38. Qa8+ Ke7 39. Ne4 Qd3 40. Nxg5 Rf5 41. Qg8 1-0.

[It has come to the Editors' notice that two more games in this match will be played, scheduled for September 17 and 24, 1989. More on these games will appear in the next issue of the Journal.] 